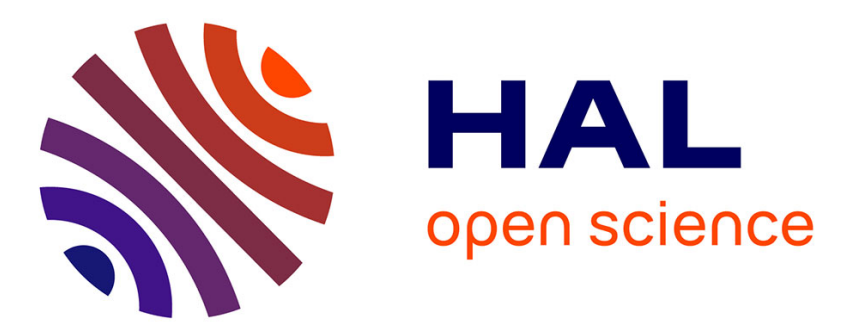

\title{
Répartitions de charges possibiliste et probabiliste pour l'analyse des incertitudes
}

Wendy Carolina Briceño Vicente, Raphaël Caire, Nouredine Hadjsaid

\section{To cite this version:}

Wendy Carolina Briceño Vicente, Raphaël Caire, Nouredine Hadjsaid. Répartitions de charges possibiliste et probabiliste pour l'analyse des incertitudes. European Journal of Electrical Engineering, 2015, 17 (3-4), pp.233 - 266. 10.3166/EJEE.17.233-266 . hal-01809965

\section{HAL Id: hal-01809965 https://hal.science/hal-01809965}

Submitted on 23 Jul 2019

HAL is a multi-disciplinary open access archive for the deposit and dissemination of scientific research documents, whether they are published or not. The documents may come from teaching and research institutions in France or abroad, or from public or private research centers.
L'archive ouverte pluridisciplinaire HAL, est destinée au dépôt et à la diffusion de documents scientifiques de niveau recherche, publiés ou non, émanant des établissements d'enseignement et de recherche français ou étrangers, des laboratoires publics ou privés. 


\section{Répartitions de charges possibiliste et probabiliste pour l'analyse des incertitudes}

\section{Wendy Carolina BRICENO VICENTE — Raphaël CAIRE - Nouredine HADJSAID}

G2ELAB, UMR 5529 INPG/UJF-CNRS

11, rue des Mathématiques BP 4638402 St Martin d'Hères Cedex, France

wendy.briceno-vicente@g2elab.grenoble-inp.fr

raphael.caire@g2elab.grenoble-inp.fr

nouredine.hadjsaid@g2elab.grenoble-inp.fr

RESUME. La connaissance de la répartition des flux de puissance à court et long terme est une donnée essentielle pour la planification, le dispatching et l'analyse des réseaux de distribution. La prise en compte de sources d'énergie alternatives dans ce calcul est devenue indispensable pour décider du plan de production optimal, de la topologie du réseau ou de la gestion des charges afin de minimiser le coût d'exploitation tout en respectant les contraintes de fonctionnement et la fiabilité des réseaux électriques. Cet article présente des stratégies pour la résolution du problème de répartition de charges en tenant compte du caractère incertain de la production de puissance décentralisée et des charges. Deux méthodes sont ainsi démontrées et comparées : la méthode probabiliste ou Simulation de Monté Carlo et la méthode possibiliste ou " arithmétique floue ».

ABSTRACT. The Power Flow studies in short and long term are essential in planning, dispatching, and the analysis of electrical networks. The consideration of renewable energy sources in the power flow computation has become indispensable to determine the optimal point of power generation of the machines, the network topology, as well as the load management plan to minimize operational costs, respecting the network constraints and maintaining the reliability of the power network. The aim of this paper is to develop a strategy of load flow considering the influence of power injection variations of distributed generation and the load consumption. Two methods are proposed and compared: the probabilistic method of Monte Carlo and the possibilistic method of the fuzzy arithmetic.

MotS-CLES: Calcul de répartition des charges, Méthode de simulation de Monté Carlo, arithmétique floue, variables aléatoires, nombre flou, intervalle arithmétique.

KEYWORDS: Power Flow, Monte Carlo method, fuzzy arithmetic, random variable, fuzzy number, interval arithmetic.

DOI:10.3199/JESA.45.1-n @ Lavoisier 2014

European Journal of Electrical Engineering $-n^{\circ} 1 / 2014,1-5$ 


\section{Introduction}

Les évolutions récentes des systèmes énergétiques ont des répercussions importantes sur les réseaux électriques, en particulier de distribution, où une large part de la production décentralisée est, ou sera, raccordée. L'incertitude de prévision de la production renouvelable ajoute une contrainte forte sur des réseaux qui ont été conçus, à l'origine, uniquement pour accueillir des consommateurs. Le réseau électrique peut se trouver proche des limites de fonctionnement sans qu'on puisse le prévoir. Pour cette raison il faut disposer de modèles qui permettent de mieux évaluer les capacités de transit du réseau dans un environnement incertain. Au cours des dernières années, de nouvelles approches de calcul de répartition des charges ont émergé. Ces approches sont basées sur des méthodes qui permettent d'inclure les incertitudes liées au problème et de les modéliser en tenant compte du caractère aléatoire ou incertain de, par exemple, certaines productions renouvelables.

La première formulation du calcul de répartition des charges probabiliste a été introduite au début des années 1970s (Borkowska, 1974), (Allan et al., 1974). Les deux principales hypothèses de cette approche sont la linéarisation et l'indépendance entre variables aléatoires. Si les charges sont des variables aléatoires indépendantes, le calcul de répartition de charges est résolu en utilisant la Transformée Rapide de Fourier. Initialement, le calcul de répartition des charges découplée linéarisé a été utilisé, puis d'autres méthodes plus détaillées ont émergé (Allan et al., 1976). On peut trouver des améliorations dans la littérature, voir les articles de (Leite da Silva et al., 1985) et (Leite da Silva et al., 1990). Toutefois, l'hypothèse d'indépendance des charges est peu réaliste. Dans les références (Allan et al., 1977), ainsi que dans (Allan et al., 1981) et (Leite da Silva et al., 1984) les distributions des charges sont normales et linéairement dépendantes.

La formulation du calcul de répartition de charges stochastique reprend les équations exactes de la répartition de charges (Dopazo et al., 1975). Dans cette analyse, la charge et la production sont traitées comme des variables aléatoires, le bruit aléatoire devient alors une distribution normale dont la covariance définie les variations des injections de puissance autour de leurs valeurs moyennes. Cette approche présente l'avantage d'utiliser à la fois le modèle détaillé et la corrélation entre les charges. Toutefois, la Simulation de Monté Carlo indique que la normalité des états du système et des sorties sont des hypothèses peu fiables (Allan et al., 1981). La méthode de moments d'ordre supérieur (troisième et quatrième) (Sauer, 1977) est une représentation plus précise de la fonction de densité de probabilité, tels que l'expansion de Gram-Charlier de la distribution de sortie (Zhang et al., 2004), ou l'orthogonalisation de Gram-Schmidt (Caramanis et al., 1982), (Bloom, 1985).

Quelques années auparavant, en 1965, L.A. Zadeh introduit la théorie des ensembles flous comme un mécanisme pour représenter le caractère vague et imprécis des concepts utilisés dans le langage commun. Ces ensembles flous ont été définis comme une extension des modèles classiques prenant en compte l'imprécision des concepts humains. Au milieu des années 70 l'extension de la notion d'ensemble logique apparaît ainsi que les premières applications aux systèmes de contrôle-commande. Cet article propose l'utilisation des ensembles 
flous pour la modélisation des incertitudes du réseau électrique avec production décentralisée. La deuxième section traite les différences entre la modélisation déterministe et la modélisation des incertitudes liées au réseau électrique.

\section{La modélisation des sources d'incertitude du réseau électrique}

La modélisation des variables électriques du réseau a été pendant de nombreuses années traitée d'une manière déterministe, tout au plus en prenant en compte l'incertitude des paramètres pour les cas les plus défavorables. L'évaluation des cas extrêmes des variables (des maxima et des minima) a été souvent considérée comme suffisante dans les études réseaux. La modélisation déterministe extrêmale représente la manière dont les variables du système sont combinées avec un modèle mathématique pour reproduire les cas extrêmes du système, sans prendre en compte des variations continues et aléatoires qui peuvent avoir lieux (Briceno Vicente, 2012). Cette modélisation considère que toutes les contraintes physiques et les actions de contrôle qui ont lieu pendant le fonctionnement du système sont fixes et il n'y a pas d'autres possibilités.

La prise en compte de toutes les valeurs possibles d'entrées fait partie de la modélisation des incertitudes. La prise en compte des variations peut potentiellement changement l'état du système. Cette modélisation stochastique permet d'explorer les états possibles du système et leurs conséquences quand les contraintes du système ne sont pas satisfaites. Les principales sources d'incertitude du réseau électrique sont :

- l'incertitude des variables d'entrée liées aux variations de la charge et à la puissance produite par les sources non-dispatchables (facteurs endogènes). L'incertitude de la charge peut varier en fonction du comportement des consommateurs (industriels, commerciaux ou résidentiels). L'incertitude de la puissance produite non-dispatchable dépend, outres les paramètres exogènes décrits dans le paragraphe suivant) d'une part de l'utilisation des machines (mode de fonctionnement, récupération de la puissance maximale), et d'autre part de la perte éventuelle d'un générateur due à une panne (disponibilité).

- l'incertitude des variables externes qui affectent les variables d'entrée comme les conditions météorologiques (facteurs exogènes).

- l'incertitude de la configuration du système. Cette incertitude est liée à la perte d'un ou de plusieurs composants du réseau suite à une défaillance. Cet état conduit à une nouvelle topologie du réseau (Alvarado, 1992).

La prise en compte des incertitudes dans la modélisation du réseau permet de quantifier l'incertitude globale liée aux entrées stochastiques du système. Cette approche s'éloigne de la technique classique d'évaluation des scénarios et des pires conditions du réseau (calculs déterministes extrémaux), pour considérer l'ensemble des états possibles du réseau. Afin de modéliser des incertitudes des variables électriques, il faut collecter des données et choisir un modèle mathématique approprié, pour évaluer leurs effets sur le réseau. Les principales approches mathématiques pour l'analyse des incertitudes des réseaux électriques quantitative et qualitative dans la littérature sont méthodes : probabilistes (Borkowska, 1974) avec 
des fonctions de densité de probabilité, ou possibilistes avec l'arithmétique des nombres flous (Sauer, 1992).

\section{La modélisation probabiliste}

L'approche probabiliste modélise l'incertitude de chaque variable d'entrée du système électrique comme une variable aléatoire qui suit une loi de probabilité. La loi de probabilité ou la fonction de densité de probabilité, modélise les valeurs que la variable peut prendre avec une expression mathématique en fonction de sa probabilité d'occurrence. La fonction de densité de probabilité est toujours liée à une fonction de répartition qui somme toutes les probabilités dues à ses valeurs possibles. Dans la littérature, deux approches probabilistes sont souvent présentées : les méthodes analytiques et les simulations stochastiques comme la méthode de Simulation de Monté Carlo.

\subsection{La méthode de Simulation de Monté Carlo}

La méthode de Simulation de Monté Carlo permet de résoudre des problèmes physiques et mathématiques à travers la simulation de variables aléatoires. C'est un outil standard pour l'analyse des systèmes complexes multidimensionnels. Elle peut être utilisée pour résoudre des problèmes stochastiques ou déterministes, mais l'application stochastique est la plus habituelle. Cette méthode est basée sur un processus itératif, raison pour laquelle, à chaque étape du processus, l'ensemble des valeurs des variables aléatoires impliquées est utilisé pour réaliser le calcul désiré, par exemple un calcul de répartition des charges déterministe. Ces valeurs sont générées en fonction des fonctions de densité de probabilité de chaque variable.

\subsection{Les types de simulation de Monté Carlo}

Les deux principales caractéristiques de la simulation Monté Carlo (MC) sont la génération de nombres aléatoires et un échantillonnage aléatoire. La méthode de MC peut être :

- Non séquentielle, aussi connue sous le nom d'échantillonnage des états, effectuent un échantillonnage aléatoire sur l'espace d'état du système pendant la période d'analyse, indépendamment de la chronologie des événements. Cette méthode ne tient pas compte des aspects temporels de la modélisation du système.

- Séquentielle simule le comportement du système en fonction du temps. La méthode construit un ensemble de séquences d'états des éléments qui composent le système, au hasard pendant la période de simulation. Elle est approprié dans les systèmes où il est nécessaire de modéliser les événements dépendants du temps ou lorsque la chronologie des événements est importante. Il y a trois types de méthodes séquentielles: synchrones, asynchrones et du temps mélangé qui diffèrent dans la génération d'événements pendant la simulation. 


\subsection{La fonction de densité de probabilité}

L'incertitude est modélisé par une variable aléatoire $X$ qui suit une fonction de densité de probabilité et définit une distribution cumulative de probabilité inversible $F_{X}(x)=P(X \leq x)$. L'échantillonnage de toutes les variables aléatoires dans les études de Simulation de Monté Carlo est fait à partir de la distribution de probabilité. Par exemple, les variations de la charge dépendent de l'heure de la journée et du jour de l'année. Ces variations peuvent être modélisées par différentes distributions et leurs probabilités d'occurrence sont montrées sur la figure 1.
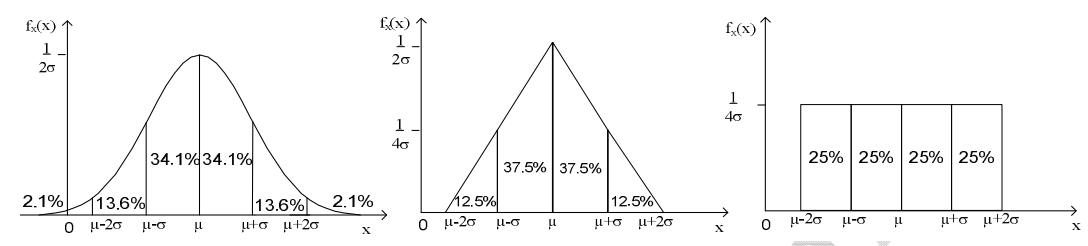

Figure 1. Les distributions de probabilités (a) normale (b) triangulaire (c) uniforme

Les fonctions de densité de probabilité présentées dans la figure 1 ont les paramètres suivants : normale $N(\mu, \sigma)$, triangulaire $T(\mu, 2 \sigma)$ ou uniforme $U(\mu \pm 2 \sigma)$, où $\mu$ est l'espérance mathématique et $\sigma$ est l'écart type.

\subsection{La précision de la méthode de simulation de Monte-Carlo}

Le processus d'échantillonnage de la méthode de simulation MC est répété itérativement jusqu'à satisfaire une marge d'erreur. Pour cette raison, il est nécessaire de déterminer le nombre d'itérations nécessaires pour atteindre un niveau de confiance d'erreur maximale attendue. L'erreur de simulation est déterminé en fonction de la taille de l'échantillon et de la précision de l'estimation à travers de l'équation suivante (Ellard et al., n.d.) :

$$
e=\frac{z}{2 \sqrt{n}}
$$

où $n$ est la taille de l'échantillon; $z$ la fonction du degré de confiance de l'estimation et $e$ la marge d'erreur, qui est la fraction pour que l'estimation soit incorrecte. Le tableau suivant contient les valeurs de $z$ pour certains niveaux de confiance typiques :

Tableau 1. Les niveaux de confiance de simulation de Monte Carlo

\begin{tabular}{|c|c|c|c|}
\hline Degré de confiance & $95,00 \%$ & $99,00 \%$ & $99,90 \%$ \\
\hline $\mathrm{z}$ & 1,96 & 2,58 & 3,29 \\
\hline
\end{tabular}


L'équation (1) et le tableau 1 ne doivent pas être utilisées pour $n \leq 30$, ou dans les cas où les probabilités soient extrêmement proches de 0 ou 1 , au moins qu'un grand nombre d'échantillons soient prévus.

\section{La modélisation possibiliste de l'incertitude}

Les ensembles flous ont été définis comme une extension des modèles classiques prenant en compte l'imprécision des concepts humains. Au milieu des années 1970 l'extension de la notion d'ensemble logique apparaît ainsi que leurs applications aux systèmes de contrôle-commande. La théorie des ensembles flous a été reconnue comme un outil potentiel pour améliorer la capacité de traitement des problèmes trop complexes ou mal définis pour être résolu par des méthodes conventionnelles. Un modèle flou peut, soit décrire un degré d'incertitude de la valeur exacte du modèle, soit donner une description linguistique de la gamme possible des valeurs basées sur des jugements humains.

\subsection{Les ensembles classiques}

La notion d'appartenance ou non d'un élément à un ensemble $A$ peut être exprimée numériquement par une fonction caractéristique $\varphi_{A}$. Cette fonction affecte à chaque élément $\mathrm{x}$ une valeur 0 ou 1 .

$$
\varphi_{A}: S \rightarrow\{0,1\} \quad \varphi_{A}(x)= \begin{cases}1 & \text { si } x \in A \\ 0 & \text { si } x \notin A\end{cases}
$$

Tout ensemble $A \subset S$ peut être défini par les couples qui composent chaque élément $x$ de l'univers et sa fonction caractéristique $\varphi$, exprimée comme suit : $A=\left\{\left(x, \varphi_{A}(x)\right): x \in S\right\}$

\subsection{Les ensembles flous ou nombres flous}

Pour modéliser de manière possibiliste une incertitude, la fonction caractéristique de l'ensemble $A$ est remplacée par une fonction d'appartenance $\mu_{A}$. La fonction d'appartenance ne prend plus uniquement les valeurs discrètes $\{0,1\}$, mais celles de l'intervalle fermé continu [0,1]. Cette fonction détermine le degré dans lequel un élément $x \in[0,1]$ (où $S$ est l'univers) appartient à l'ensemble $A$.

$$
\mu_{A}: S \rightarrow[0,1] \quad \mu_{A}(x) \in[0,1]
$$

où $\mu_{A}(x)$ donne le degré d'appartenance de la variable $x$ dans l'intervalle continu $[0,1]$ vers la condition qui constitue l'ensemble. Lorsque $\mu_{A}(x)=0$, l'élément n'appartient pas à l'ensemble $A$, et quand $\mu_{A}(x)=1$ il lui appartient 
entièrement, toute valeur intermédiaire va traduire une imprécision/incertitude de la détermination.

\subsection{Les propriétés des nombres flous}

La fonction d'appartenance doit satisfaire les trois propriétés suivantes :

- la fonction est normalisée, le maximum de la fonction est 1. Le noyau est la valeur ou intervalle des valeurs pour lesquelles la fonction vaut 1.

- la fonction est convexe, tous les niveaux de l'ensemble, appelés aussi coupes alpha (voir section 4.4), sont des intervalles fermés.

- la fonction est toujours continue et bornée.

Les nombres flous composées par une partie réelle et imaginaire doivent accomplir les conditions d'appartenance de ses parties réelle et imaginaire (Klir et al., 1995).

\subsection{Les coupes alpha des nombres flous}

Les coupes alpha de l'ensemble flou $A_{\alpha}$ sont constitués par les éléments dont le degré d'appartenance dépasse ou atteint le seuil alpha $\mu_{A}(x) \geq \alpha$ avec $\alpha \in[0,1]$ et détermine un intervalle de variation fermé pour chaque valeur $\alpha$.

$$
\varphi_{A \alpha}(x)= \begin{cases}1 & \text { si } \mu_{A}(x) \geq \alpha \\ 0 & \text { autrement }\end{cases}
$$

Les coupes alpha sont particulièrement utiles dans l'arithmétique floue comme on le verra dans la section suivante.

\subsection{Les nombres flous trapézoüdaux}

Les fonctions d'appartenance trapézoïdales sont déterminées par quatre paramètres qui se réfèrent aux points $a_{1}, a_{2}, a_{3}$ et $a_{4}$ de la fonction d'appartenance. La forme exacte de la fonction d'appartenance trapézoïdale est définie par l'équation (Hanss, 2005):

$$
\mu_{A}(x)= \begin{cases}\frac{x-a_{1}}{a_{2}-a_{1}}, & \text { pour } a_{1} \leq x \leq a_{2} \\ 1 & \text { pour } a_{2} \leq x \leq a_{3} \\ \frac{a_{4}-x}{a_{4}-a_{3}}, & \text { pour } a_{3} \leq x \leq a_{4} \\ 0 & \text { sinon }\end{cases}
$$

où $a_{1}, a_{4}$ sont les valeurs pour les extrêmes $\alpha=0, a_{2}, a_{3}$ les valeurs extrêmes $\alpha=1$ avec $a_{1} \leq a_{2} \leq a_{3} \leq a_{4}$. La fonction d'appartenance peut être aussi écrite en termes des intervalles de confiance, donc $\alpha$-coups avec l'équation suivant: 


$$
A_{\alpha}=\left[a_{1}+\alpha\left(a_{2}-a_{1}\right), a_{4}-\alpha\left(a_{4}-a_{3}\right)\right], \forall \alpha \in[0,1]
$$

Ce modèle à quatre paramètres peut également être utilisé pour représenter les fonctions d'appartenance triangulaire $\left(a_{2}=a_{3}\right)$ ou rectangulaire $\left(a_{1}=a_{2}\right.$ et $\left.a_{3}=a_{4}\right)$.
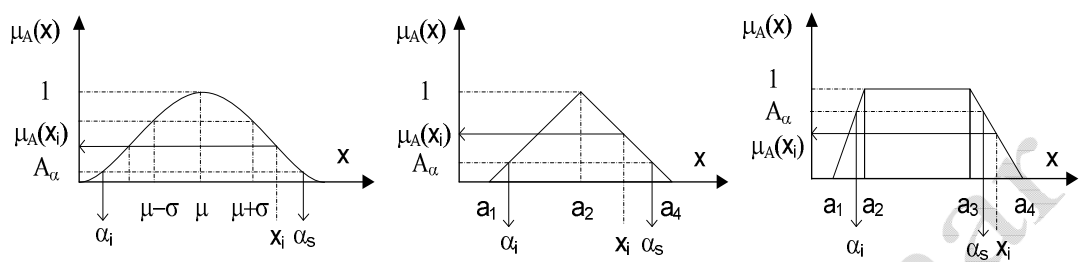

Figure 2. Les nombres flous (a) gaussien, (b) triangulaire et (c) trapézoïdal

Les valeurs des coefficients $a_{1}, a_{2}, a_{3}$ et $a_{4}$ peuvent être déterminés à partir du principe de cohérence de la transformation possibilité-probabilité, voir section 5 où des méthodes de passage entre les deux arithmétiques (probabilistes et possibilistes) sont présentées.

\subsection{Les opérations arithmétiques sur les nombres flous}

Les Règles pour les opérations arithmétiques entre les nombres flous peuvent être déduites du principe d'extension de (Zadeh, 1999). S'il existe deux nombres flous $A$ et $B$ définis par leurs quadruples, tels que : $A=\left[a_{1}, a_{2}, a_{3}, a_{4}\right]$ et $B=\left[b_{1}, b_{2}, b_{3}, b_{4}\right]$.

$$
\begin{aligned}
& A+B=\left[a_{1}+b_{1}, a_{2}+b_{2}, a_{3}+b_{3}, a_{4}+b_{4}\right] \\
& A-B=\left[a_{1}-b_{4}, a_{2}-b_{3}, a_{3}-b_{2}, a_{4}-b_{1}\right] \\
& A \times B=\left[a_{1} \times b_{1}, a_{2} \times b_{2}, a_{3} \times b_{3}, a_{4} \times b_{4}\right] \\
& A \div B=\left[a_{1} \div b_{4}, a_{2} \div b_{3}, a_{3} \div b_{2}, a_{4} \div b_{1}\right]
\end{aligned}
$$

Les opérations arithmétiques entre nombres flous sont évaluées pour chacune des valeurs extrêmes de chaque intervalle $\alpha=0$ et $\alpha=1$.

\section{La transformation possibiliste-probabiliste}

L'une des questions les plus controversées en matière de modélisation d'incertitude est la relation entre la théorie des probabilités et celle des ensembles flous. La littérature se rapportant à ce débat peut être trouvée dans (Dubois, 1993). Ces auteurs proposent une transformation optimale pour que les pertes d'informations soient les plus faibles possibles. Ainsi, une variable incertaine peut être associée à une distribution de probabilité ou à une distribution de possibilité. 
Le concept du principe de cohérence entre la possibilité et la probabilité (PCPP) a été proposé par (Zadeh, 1978) pour parvenir à un compromis entre la possibilité et la probabilité de l'information. Ce compromis démontre que la diminution de la possibilité d'un événement tend à réduire sa probabilité, mais non réciproquement. Soit $U$ l'univers, $P$ et $\Pi$ la mesure de probabilité et possibilité associée à la distribution de probabilité et de possibilité $p$ et $\pi$. La transformation est possible si les conditions suivantes sont vérifiées :

- le principe de cohérence :

$$
P(A) \leq \Pi(A), \forall A \subset X
$$

- le principe de préférence :

$$
\left\{\begin{array}{l}
p(a)<p(b) \Rightarrow \pi(a)<\pi(b) \\
p(a)=p(b) \Rightarrow \pi(a)=\pi(b)
\end{array}, \forall a, b \in X\right.
$$

La mesure de la cohérence entre la possibilité et la probabilité est exprimée par l'équation suivante (Hanss, 2005) :

$$
C_{z}(\pi, p)=\sum \pi(x) \cdot p(x)=\int_{x \in U} \pi(x) \cdot p(x)
$$

où $C_{z}$ est la valeur qui montre le degré de cohérence de la transformation entre la fonction de densité de probabilité et sa distribution de densité de possibilité. Elle implique le degré de maintien de l'information au cours de la transformation.

Des techniques d'optimisation sous contraintes ont été développées pour obtenir des fonctions de probabilité à partir des fonctions de possibilité (Civanlar, 1986) qui correspondent à un degré déterminé par la méthode d'optimisation utilisée cidessous :

$$
\begin{gathered}
\min \frac{1}{2} \int_{-\infty}^{+\infty} \mu^{2}(x) \cdot d x \\
C_{z}(\pi, p)-\int_{-\infty}^{+\infty} \pi(x) \cdot p(x) \cdot d x \leq 0
\end{gathered}
$$

où $\mu(x)$ est la moyenne dérivée de la fonction de possibilité, $C_{z}$ le degré de cohérence de cette transformation. $p(x)$ la possibilité que la valeur réelle moyenne soit situé dans l'intervalle moyen défini par la fonction de possibilité moyenne. La borne inférieure de $C_{z}$ doit satisfaire le lemme suivant pour pouvoir trouver la valeur minimale de la transformation. 
10 EJEE. Volume $1-\mathrm{n}^{\circ} 1 / 2014$

Par exemple, une fonction de probabilité normale standard $p(x): N(0,1)$ est transformée en fonction de possibilité $\mu(x)$ qui dépend d'un paramètre $S$. La valeur de $S$ détermine le degré de cohérence $C_{z}$ afin de démontrer le processus.

$$
\begin{gathered}
\mu(x)=\left\{\begin{array}{l}
e^{\frac{S^{2}-x^{2}}{2}},|x|>S \\
1,|x|<S
\end{array}\right. \\
C=\sqrt{\frac{1}{2}}\left[e^{\frac{S^{2}}{2}} \cdot \int_{-\infty}^{+\infty} e^{-x^{2}} \cdot d x+\int_{0}^{S} e^{-\frac{x^{2}}{2}} \cdot d x\right]
\end{gathered}
$$

La valeur $C$ peut être évaluée en utilisant une technique d'analyse numérique. Lorsque la valeur de $C$ est supérieure ou égale à $2^{-1 / 2}$, la fonction de possibilité dérivée de la densité normale sera en conformité avec le PCPP.

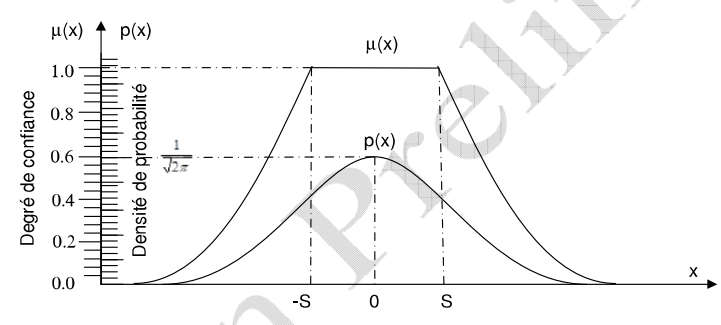

Figure 3. Les foncions de densité de probabilité p et distribution de possibilité $\mu$

\section{Le calcul de répartition de charges}

Le calcul de répartition des charges est l'un des outils le plus largement utilisé pour l'exploitation (plan de production économiquement optimal, réglage en tension optimisé, entre autres), et la planification des réseaux électriques (en vérifiant les contraintes des composants électriques tels que : compensateurs shunt, prises des transformateurs, ceci avec différentes topologies). La répartition de charges classique est une méthode déterministe, avec données d'entrée constantes. Elle fournit les valeurs en régime permanent (fréquence nominale) des variables électriques de la tension à chaque nœud (amplitude et angle) ainsi que les puissances (active et réactive) transitant dans les lignes d'un réseau pour un scénario spécifié des charges et des conditions d'exploitation. La modélisation des éléments principaux du réseau dans l'algorithme comprend :

- les lignes ou câble souvent représentées, pour les réseaux de distribution par une impédance série, hypothèse suffisamment précise pour des lignes courtes. 
- les transformateurs souvent représentés par une impédance shunt (qui représente le courant magnétisant et les pertes fer) et une impédance série (qui représente l'inductance de fuite et les pertes Joule).

- les charges électriques souvent représentées par une combinaison linéaire des trois sous partie suivantes : courant constant, puissance constante, ou impédance constante. Les équations (12) et (13) présentent la modélisation exponentielle de la charge (Kundur, 1994).

$$
\begin{aligned}
& P=P_{n} \times\left(V / V_{n}\right)^{k_{1}} \\
& Q=Q_{n} \times\left(V / V_{n}\right)^{k_{2}}
\end{aligned}
$$

où $P_{n}$ et $Q_{n}$ sont respectivement les puissances active et réactive à la tension nominale $V_{n}$ du nœud $n$ et $V$ la tension du nœud de la charge. Pour les charges à puissance constante : $k_{1}=k_{2}=0$, pour les charges à courant constant : $k_{1}=k_{2}=1$ et pour les charges à impédance constante: $k_{1}=k_{2}=2$. Dans la suite du développement de l'algorithme et compte tenu des données réseau disponibles pour l'étude, la modélisation à puissance constante a été retenue.

\subsection{L'algorithme du calcul de répartition de charges}

Le réseau de distribution est principalement exploité en structure radiale ou faiblement maillée, en régime polyphasé-déséquilibré (charges monophasés, biphasés et triphasé en déséquilibre), un grand nombre de branches et de nœuds, une non-transposition des conducteurs qui conduit à des couplages mutuels différents entre les lignes et avec un ratio résistance/réactance élevé. Ces caractéristiques ont tendance à rendre peu efficaces des méthodes traditionnelles de calcul de répartition des charges (type Gauss Seidel ou Newton-Raphson) largement utilisées dans les réseaux de transport (plus souvent modélisé représenté par un réseau équilibré (Briceño Vicente, 2012)).

L'algorithme retenu pour le calcul de répartition de charge (Teng, 2003) prend en compte les avantages des caractéristiques topologiques des réseaux de distribution et permet une utilisation simple des règles arithmétiques (voir section 4.6) des nombres flous. Deux matrices sont employées, la matrice BIBC (en Anglais Bus Injection Branch Current) et la matrice $B C B V$ (en Anglais Branch Current Bus Voltage). Il peut être également utilisé dans les réseaux de distribution faiblement maillés. La méthode retenue est très souvent plus précise et plus efficace, du fait de l'écriture matricielle que les autres méthodes conventionnelles dans le cas les réseaux de distribution. 


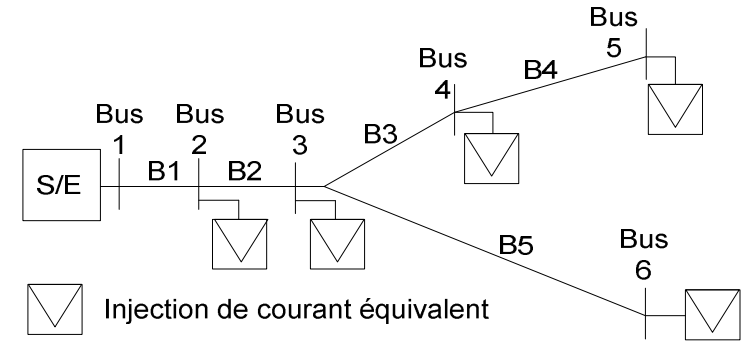

Figure 4. Réseau de distribution radial avec un poste source.

Le terme équivalent de l'injection de courant par phase à l'itération $k$ est dérivé de la puissance consommée sur le nœud i qui est exprimé par :

$$
\begin{gathered}
S_{i-\text { phase }}=\left(P_{i-\text { phase }}+j Q_{i-\text { phase }}\right), i=1, \ldots, N \\
I_{i-\text { phase }}{ }^{k}=\left(S_{i-\text { phase }} / V_{i-\text { phase }} k\right)^{*}
\end{gathered}
$$

où $S_{i-p h a s e}$ est la puissance apparente consommée par la charge de l'une des trois phases (a, b, ou c), composée de $P_{i-\text { phase }}$ et $Q_{i-\text { phase }}$ qui sont respectivement les puissances active et réactive de la charge par phase, $N$ est le nombre de charges $V_{i-p h a s e}{ }^{k}$ et $I_{i-p h a s e}^{k}$ sont respectivement la tension du nœud et l'équivalent d'injection de courant du nœud par phase à l'itération $k$.

Un réseau de distribution est illustré à la figure 4. Les injections de puissance peuvent être converties en injections de courant équivalent par l'équation 16. La relation entre les injections de courant de nœud et les courants de branche est déterminée par la loi des nœuds ou de courant de Kirchhoff. Les courants des branches peuvent alors être formulés en fonction des différents courants injectés aux nœuds en aval de cette branche. Par conséquent, cette relation peut être exprimée comme :

$$
\left[\begin{array}{l}
B_{1 a b c} \\
B_{2 a b c} \\
B_{3 a b c} \\
B_{4 a b c} \\
B_{5 a b c}
\end{array}\right]=\left[\begin{array}{ccccc}
\tilde{1} & \tilde{1} & \tilde{1} & \tilde{1} & \tilde{1} \\
\tilde{0} & \tilde{1} & \tilde{1} & \tilde{1} & \tilde{1} \\
\tilde{0} & \tilde{0} & \tilde{1} & \tilde{1} & 0 \\
\tilde{0} & \tilde{0} & \tilde{0} & \tilde{1} & 0 \\
\tilde{0} & \tilde{0} & \tilde{0} & \tilde{0} & \tilde{1}
\end{array}\right]\left[\begin{array}{c}
I_{2 a b c} \\
I_{3 a b c} \\
I_{4 a b c} \\
I_{5 a b c} \\
I_{6 a b c}
\end{array}\right] \rightarrow[\mathrm{B}]_{a b c}=[B I B C][I]_{a b c}
$$

où $B I B C$ (en Anglais Bus Injection Branch Current) est la matrice qui exprime la relation entre le courant injecté au nœud et le courant de branche, 
$B_{i a b c}=\left[\begin{array}{lll}B_{i a} & B_{i b} & B_{i c}\end{array}\right]^{T}, i=1,2, \ldots, 5$ est le vecteur des injections de courants de branche $i, I_{i a b c}=\left[\begin{array}{lll}I_{i a} & I_{i b} & I_{i c}\end{array}\right]^{T}, i=2,3, \ldots, 6$ est la vecteur des courants de nœud $i$. Les symboles $\tilde{0}, \tilde{1}$ représentent les vecteurs $\left[\begin{array}{lll}0 & 0 & 0\end{array}\right]^{T}$ et $\left[\begin{array}{lll}1 & 1 & 1\end{array}\right]^{T}$. Pour le cas équilibre $\tilde{0}, \tilde{1}$ deviennent simplement 0,1 . La matrice $B I B C$ est triangulaire supérieure ne contient que des valeurs de 0 et 1 .

La tension de nœud peut être exprimée comme une fonction des courants de branche, des paramètres de ligne, et de la tension de référence au niveau du poste source (HTB/HTA). La relation entre les courants de branche et les tensions de nœud est obtenue par les équations 17 et 18.

$$
\begin{aligned}
{\left[\begin{array}{l}
V_{1 a b c} \\
V_{1 a b c} \\
V_{1 a b c} \\
V_{1 a b c} \\
V_{1 a b c}
\end{array}\right]-\left[\begin{array}{l}
V_{2 a b c} \\
V_{3 a b c} \\
V_{4 a b c} \\
V_{5 a b c} \\
V_{6 a b c}
\end{array}\right]=} & {\left[\begin{array}{lllll}
\tilde{Z}_{12 a b c} & 0 & 0 & 0 & 0 \\
\tilde{Z}_{12 a b c} & \tilde{Z}_{23 a b c} & 0 & 0 & 0 \\
\tilde{Z}_{12 a b c} & \tilde{Z}_{23 a b c} & \tilde{Z}_{34 a b c} & 0 & 0 \\
\tilde{Z}_{12 a b c} & \tilde{Z}_{23 a b c} & \tilde{Z}_{34 a b c} & \tilde{Z}_{45 a b c} & 0 \\
\tilde{Z}_{12 a b c} & \tilde{Z}_{23 a b c} & 0 & 0 & \tilde{Z}_{36 a b c}
\end{array}\right]\left[\begin{array}{l}
B_{1 a b c} \\
B_{2 a b c} \\
B_{3 a b c} \\
B_{4 a b c} \\
B_{5 a b c}
\end{array}\right] } \\
& {[\Delta \mathrm{V}]_{a b c}=[B C B V]_{a b c}[B]_{a b c} }
\end{aligned}
$$

où $B C B V$ (en Anglais Branch Current Bus Voltage) est la matrice qui représente la relation entre les courants de branches et les tensions des nœuds, $V_{i_{a b c}}=\left[\begin{array}{lll}V_{i_{a}} & V_{i b} & V_{i_{c}}\end{array}\right]^{T}, i=2,3, \ldots, 6$ est la tension triphasée du nœud $i$, et $Z_{i j}{ }_{a b c}$ est l'impédance de ligne triphasée entre le nœud $i$ et le nœud $j$.

Les matrices $B I B C$ et $B C B V$ sont élaborées en fonction de la structure topologique du réseau de distribution. En combinant les équations 19 et 20, la relation entre ces deux matrices peut être exprimée comme :

$$
\begin{gathered}
{[\Delta V]_{a b c}=[B C B V][B I B C][I]_{a b c}} \\
{[\Delta V]_{a b c}=[D L F][I]_{a b c}}
\end{gathered}
$$

où $D L F$ représente la matrice produit de $B C B V$ par $B I B C$. La solution du calcul de répartition de charges est obtenue par une résolution itérative, de l'équation 20. La tension est calculée grâce à l'équation de récurrence 21 et suivant le critère d'arrêt de l'équation 22.

$$
\begin{gathered}
\left.\mid \mathrm{V}^{\mathrm{k}+1}\right\rfloor_{a b c}=\left\lfloor\mathrm{V}^{0}\right\rfloor_{a b c}+\left\lfloor\Delta \mathrm{V}^{\mathrm{k}+1}\right\rfloor_{a b c} \\
\left\lfloor\Delta \mathrm{V}^{\mathrm{k}+1}\right\rfloor_{a b c}-\left\lfloor\Delta \mathrm{V}^{\mathrm{k}}\right\rfloor_{a b c}<\mathcal{E}
\end{gathered}
$$

où $\varepsilon$ est la précision désirée sur la chute de tension. La seule matrice nécessaire pour la résolution du calcul de répartition de charges est la matrice $D L F$. En 
conséquence, la méthode proposée peut économiser un temps de calcul considérable. L'algorithme utilisé peut être résumé par la figure suivante :

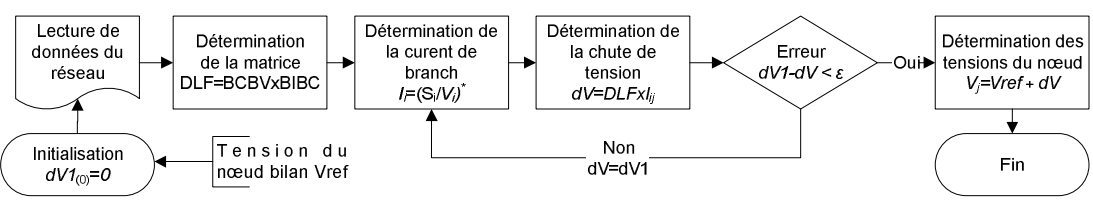

Figure 5. Algorithme de la répartition de charges pour des réseaux radiaux.

\subsection{Les incertitudes dans les calculs de répartition de charges}

Les incertitudes affectent les résultats du calcul de répartition des charges. La modélisation des incertitudes des variables électriques a été mise en œuvre, dans cette étude, avec l'approche probabiliste (par leur fonction de densité de probabilité avec une simulation de Monte-Carlo) et avec l'approche possibiliste (par leur fonction d'appartenance et les outils de l'arithmétique floue). Ces fonctions sont obtenues à partir de l'analyse des mesures déterministes faite sur les éléments d'incertitude. Le modèle présenté dans ce papier suppose que le système triphasé peut être représenté par son équivalent monophasé direct. Les principales sources d'incertitude à prendre en compte dans le calcul de répartition de charges sont :

- les variations des paramètres de ligne comme conséquence des variations de la température et des erreurs d'estimation des paramètres des lignes.

- les variations de tension au poste source (HTB/HTA) dues aux variations de tension du réseau de répartition et du régleur en charge.

- les variations de la demande, comme conséquence des activités liées à la consommation de puissance par la charge (demande imprévisible).

- les variations du facteur de puissance des charges par l'action des compensateurs de puissance réactive.

- l'intermittence des GEDs qui dépendent des conditions météorologiques.

\subsection{Proposition d'un modèle des incertitudes de la charge}

La modélisation des charges est une tâche complexe. La charge est le paramètre le plus incertain du réseau et elle varie constamment. La complexité de sa modélisation est due à l'existence de cycles de charge journaliers et annuels, qui varie, de plus, suivant la segmentation des clients. En général, la valeur la plus intéressante pour le planificateur (en charge du dimensionnement - ou planification - du réseau) est la demande de pointe annuelle, car elle détermine les besoins en capacité pour le parc de production mais également pour les contraintes supportées par le réseau. D'autre part, pour la comparaison économique, il est nécessaire de calculer les pertes annuelles du système et leur coût associé. Parmi les facteurs fondamentaux qui influencent la demande, on peut citer : 
- La température qui a un impact différent d'un pays à l'autre en fonction de la saison et affecte la charge. En outre, les charges résidentielles sont des charges très thermosensibles pendant l'hiver, dans certains pays et pendant l'été dans d'autres pays.

- Le facteur économique : qui affecte la charge de pointe annuelle à long terme, ainsi que le facteur démographique et le niveau de l'activité industrielle de la région. Ces paramètres définissent l'augmentation ou la baisse de la charge du réseau. Ils doivent être pris en compte dans la prévision de charge à long terme, qui est l'un des points de départ pour les études de planification du réseau.

- D'autres sources d'incertitude : qui sont liées aux facteurs temporels et météorologiques. Les facteurs temporels proviennent des variations saisonnières, des cycles de charges hebdomadaires, quotidiens, et de l'activité économique (cf. pendant jours fériés). Le modèle probabiliste des incertitudes de la charge à court terme est déterminé par les facteurs déjà mentionnés plus haut.

La construction du modèle retenu agrégé de la charge est faite à partir de données horaires mesurées à partir d'un poste source de $33 \mathrm{kV} / 110 \mathrm{kV}$, pendant un an avec des intervalles de temps de 10 minutes (AESO, 2011). Les variations saisonnières de la charge par rapport à la moyenne journalière et hebdomadaire sont représentées à la figure 6. Des différences existent entre les pics des jours ouvrables et les pics du week-end. Le comportement des données de la charge est donc décrit par une variable aléatoire et sa fonction de densité de probabilité. Les données annuelles de variation de la charge de la figure 7 sont soumises à une analyse détaillée.
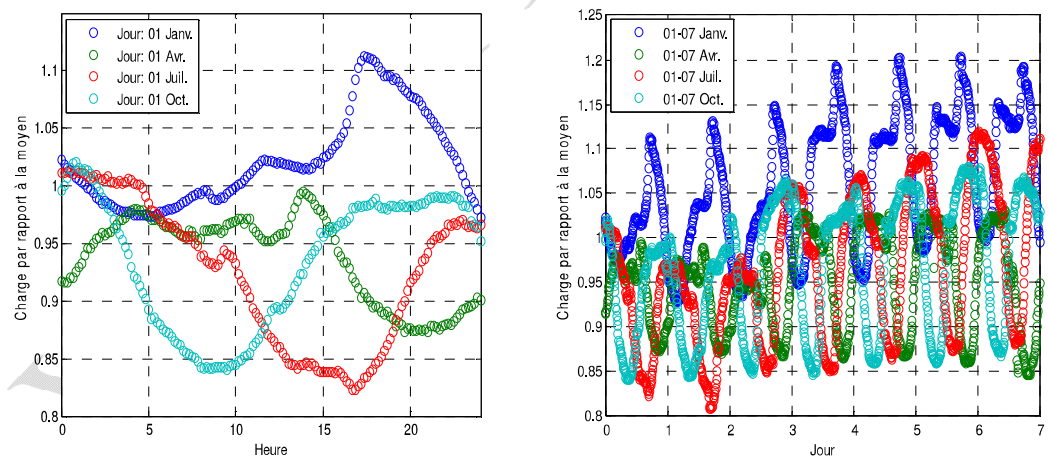

Figure 6. Variation de la charge (a) journalière (b) hebdomadaire par saison. 


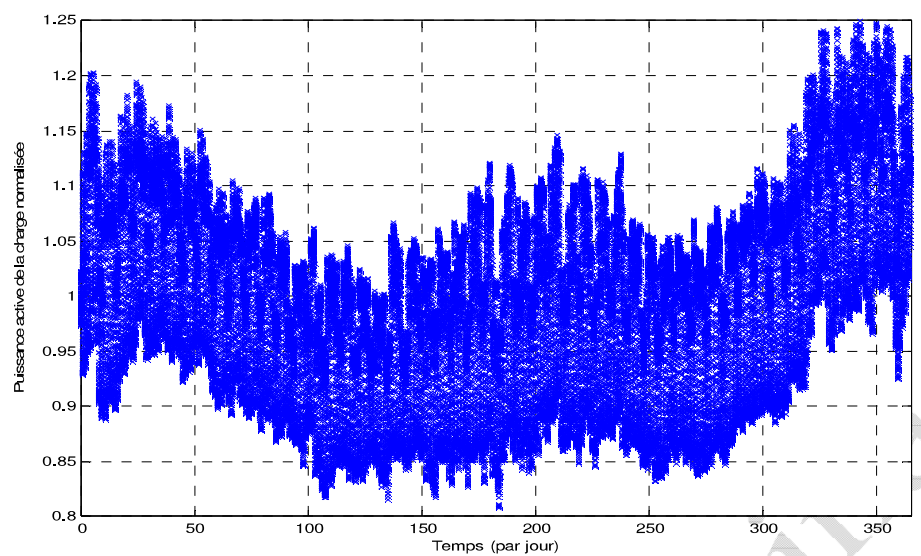

Figure 7. Variation de la charge annuelle par rapport à la moyenne.

La figure 8 montre la fonction de densité de probabilité ajustée avec MatLab, ainsi que les histogrammes de données réelles de la charge annuelle. L'histogramme montre la fréquence avec laquelle un intervalle des valeurs de donnés se répète pendant le temps d'étude.

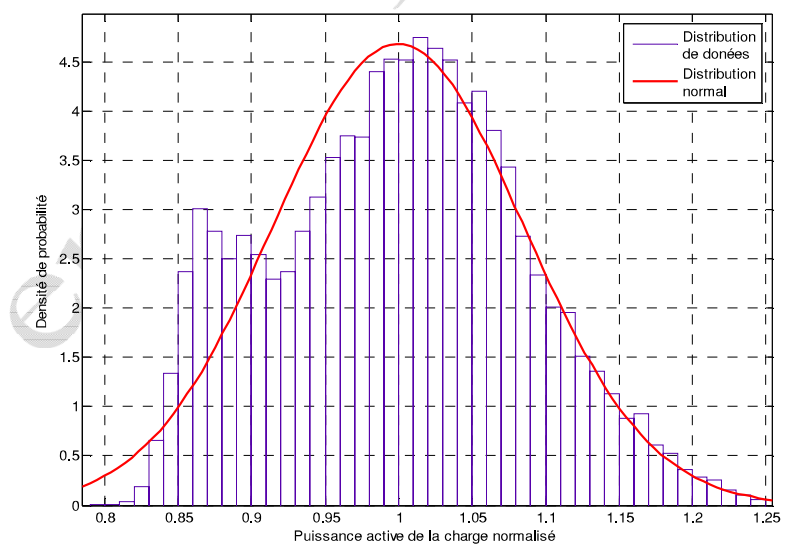

Figure 8. Fonction de densité de probabilité normale retenue et histogramme de la charge. 
L'histogramme des données de la figure 8 est bien représenté par la distribution normale, malgré l'existence d'un maximum local de la charge est à 0,85 de sa puissance moyenne. Les variations de la charge sont décrites par une distribution de type normale par l'équation :

$$
f_{x}(x)=\frac{1}{\sigma \sqrt{2 \pi}} e^{-\frac{(x-\mu)^{2}}{2 \sigma^{2}}}
$$

où $\mu$ est la moyenne et $\sigma$ l'écart type. Selon le test de vraisemblance les données correspondent avec une précision de 95\% la fonction de densité de probabilité est caractérisée par $\mu_{\%}=99,999 \pm 0,037$ et $\sigma_{\%}=8,510 \pm 0,026$, le symbole \pm indique l'erreur de l'approximation de ces deux paramètres.

\subsection{Proposition d'un modèle pour la génération éolienne}

La source primaire d'énergie d'une éolienne est la vitesse du vent, qui dépendent de facteurs tels que les saisons, le moment de la journée, la localisation et des conditions climatiques, entre autres. La vitesse du vent rend très variable la production de puissance. Le modèle utilisé pour la génération éolienne prend comme point de départ des échantillons de la vitesse du vent annuel. La modélisation de ces données a été réalisée avec une fonction de densité de probabilité.

La turbine éolienne fait la conversion d'énergie cinétique en énergie mécanique, et enfin en énergie électrique au travers du générateur. La puissance nominale d'une éolienne est la puissance maximale du générateur installé. La puissance transmise au générateur par la turbine est limitée à cette valeur grâce au système de contrôle. Ce dernier limite l'énergie cinétique récupérée souvent par l'action sur l'angle d'incidence du vent sur les pâles. Il faut veiller à ce que cette puissance ne soit pas dépassée par vents forts.

\subsubsection{Détermination de la fonction de distribution de vent}

Les données de la vitesse du vent sont généralement obtenues à partir d'une rose des vents ou d'un histogramme à partir des mesures du vent sur une période de temps longue. Le calcul de la fonction de probabilité de la vitesse du vent est donc fait avec son histogramme des données, (cf. dans la section précédente pour distribution de probabilités de la charge). Les variations de la vitesse du vent sont décrites par une distribution de type Weibull par l'équation :

$$
f_{v}(v)=\frac{\beta}{\alpha} *\left(\frac{v}{\beta}\right)^{\beta-1} * e^{-\left(\frac{v}{\alpha}\right)^{\beta}}
$$

où $v$ représente la vitesse du vent, $\alpha$ le facteur d'échelle et $\beta$ le facteur de forme. Généralement, le facteur d'échelle $\alpha$ est compris dans l'intervalle $\alpha \in(1,10)$. 
Le facteur $\beta$ est lié à la vitesse moyenne du vent, une valeur élevée est favorable pour la production d'énergie.

Les données réelles annuelles de la vitesse du vent dans une région de France ont été représentées par leur histogramme. Les fonctions de densité de probabilité et les fonctions cumulatives ont été approchées par une distribution Weibull, à partir de l'histogramme de données réelles de la vitesse du vent dans la figure 9.
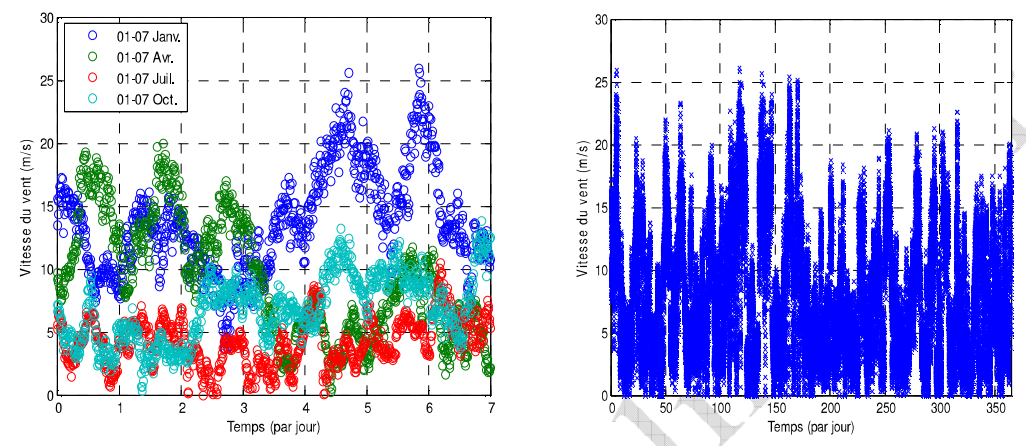

Figure 9. Variation de la vitesse du vent (a) hebdomadaire (b) annuelle

Le test de vraisemblance a été appliqué aux données de l'histogramme avec une précision de $95 \%$. La fonction de densité de probabilité ajustée correspond à une fonction de type Weibull avec des valeurs $\alpha=8,494 \pm 0,028 \mathrm{~m} / \mathrm{s}$ et $\beta=1,487 \pm 0,005 \mathrm{~m} / \mathrm{s}$, le symbole \pm indique l'erreur maximale de l'approximation.

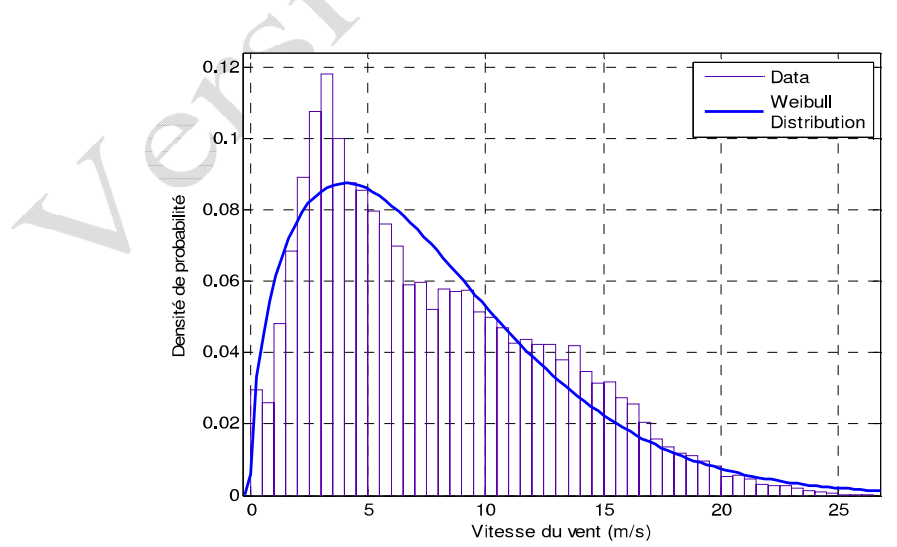

Figure 10. Fonction de densité de probabilité et histogramme de données du vent 


\subsubsection{Le modèle de l'aérogénérateur}

La courbe de puissance éolienne est déterminée par l'efficacité aérodynamique de la turbine, de la transmission mécanique, de la technologie de conversion électromécanique, de la hauteur de l'éolienne et de la vitesse du vent sur le site. Les données électriques typiques fournies par un fabricant d'éolienne sont la puissance maximale, la vitesse de démarrage, la vitesse nominale, et la vitesse de coupure. Les éoliennes peuvent produire aussi de la puissance réactive en régime permanent en fonctionne du facteur de puissance qui varie souvent de $95 \%$ à $100 \%$. Grâce à ces données, la courbe de puissance de sortie peut être extraite, elle permet de calculer la puissance de sortie de l'éolienne pour n'importe quelle vitesse du vent.

Par exemple, la courbe de puissance de la turbine Ris $\varnothing$ DTU modèle NM80 de puissance nominale de $2 \mathrm{MW}$ de la figure 11 varie sur la plage de vitesse de vent de $0 \mathrm{~m} / \mathrm{s}$ à $27 \mathrm{~m} / \mathrm{s}$. Le facteur de puissance nominal $(\cos \varphi)$ de l'éolienne est $95 \%$.

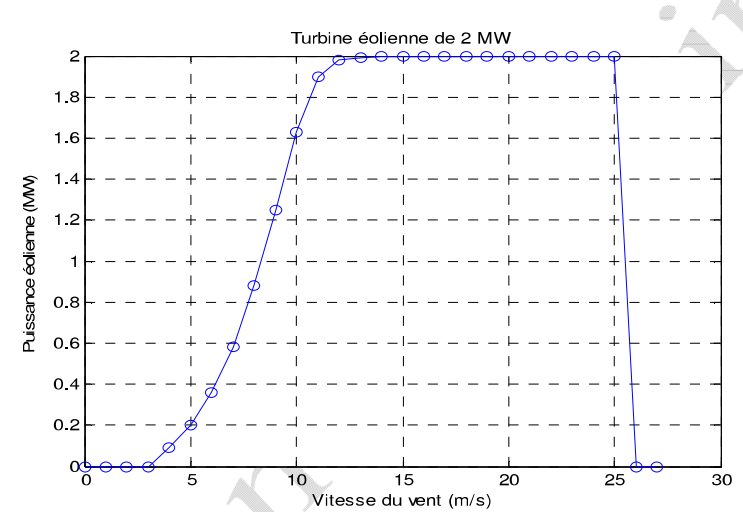

Figure 11. Fonction de transfert vitesse du vent et puissance de sortie éolienne

La courbe de puissance de l'éolienne en fonction de la vitesse du vent peut être approximée et linéarisée par parties à partir de la courbe réelle de puissance. Le modèle de la figure 11 peut ainsi être décrit par une fonction en trois parties : l'une qui représente les valeurs pour lesquelles il n'y a pas de génération de puissance, l'autre pour laquelle la puissance générée varie linéairement en fonction de la vitesse du vent et enfin un intervalle de valeurs dans lequel la puissance produite par la turbine est constante et égale à la puissance nominale de la turbine. Ce modèle de courbe de puissance de la turbine est indiqué dans l'équation suivante :

$$
P_{w}=\left\{\begin{array}{llc}
0 & \text { pour } & 0 \leq v \leq V_{R} \\
\left(\frac{P_{R}}{V_{R}-V_{C}}\right)\left(v-V_{C}\right) & \text { pour } & V_{C} \leq v \leq V_{R} \\
P_{R} & \text { pour } & V_{R} \leq v \leq V_{F}
\end{array}\right.
$$


où $P_{R}$ est la puissance maximale, $V_{C}$ vitesse du vent au démarrage, $V_{R}$ vitesse du vent nominale, $V_{F}$ vitesse maximale pour laquelle la turbine est arrêtée pour des raisons de dimensionnement et de sécurité. Le modèle de la turbine de la puissance de sortie en fonctionne de la vitesse du vent utilise les paramètres suivants : $V_{C}=$ $5 \mathrm{~m} / \mathrm{s}, V_{R}=12,5 \mathrm{~m} / \mathrm{s}$ et $V_{F}=25 \mathrm{~m} / \mathrm{s}$.

\subsubsection{Le modèle de la puissance de sortie éolienne}

La puissance de sortie éolienne est obtenue à partir de l'échantillonnage de la vitesse du vent et de la courbe de puissance de l'équation 25 . La fonction de densité de probabilité de la puissance éolienne est montrée dans la figure ci-dessous.
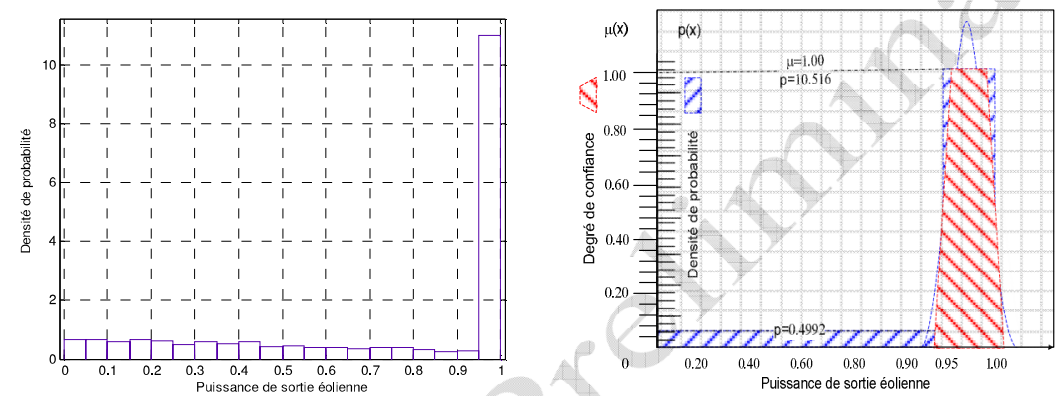

Figure 12. (a) Fonction de densité de probabilité (b) Fonction d'appartenance floue de la puissance éolienne

La fonction de densité de probabilité de la puissance éolienne produite peut être approchée par une distribution normale $N(\mu, \sigma)=N(0,975,0,025)$, voir Figure 12.b. Le modèle flou de la production d'énergie éolienne a été déterminé par une fonction d'appartenance $\mu_{A}(x)$ trapézoïdale, comme il a été fait pour la demande d'énergie. Les extrêmes de cette fonction trapézoïdale sont définis par les quatre valeurs suivantes : $\left(a_{1} ; a_{2} ; a_{3} ; a_{4}\right)=(0,95 ; 0,97 ; 0,98 ; 1,00)$.

La fonction d'appartenance floue équivalente a été obtenue en appliquant l'équation (11) sur la section de puissance de sortie éolienne comprise sur l'intervalle de 0,97 à 0,975 de la puissance nominale. En appliquant l'équation (11) de la transformation possibilité-probabilité pour la valeur $S=0,05$ on obtient $C=0,89$ ainsi la condition est supérieure ou égale à $2^{-1 / 2}$, est accomplie.

\subsection{Les algorithmes pour la répartition de charges probabiliste et possibiliste}

Les algorithmes probabilistes (simulation de Monte-Carlo) et possibilistes (arithmétique floue) s'appuient sur un calcul de répartition des charges déterministe 
présenté à la section 6.1. Les données des sources d'incertitude liées aux variables électriques du réseau, sont modélisées comme variables aléatoires puis les nombres flous sont déterminés en utilisant la transformation du PCPP. Les principales différences entre les deux algorithmes sont : le nombre d'itérations requises dont a besoin chaque méthode pour obtenir une certaine précision et le temps pour résoudre le problème. Les deux algorithmes sont montrés sur la figure 13 et montrent la procédure générale pour le traitement des incertitudes. Ces algorithmes peuvent être utilisés avec autres études du réseau comme le calcul de courants de court-circuit.

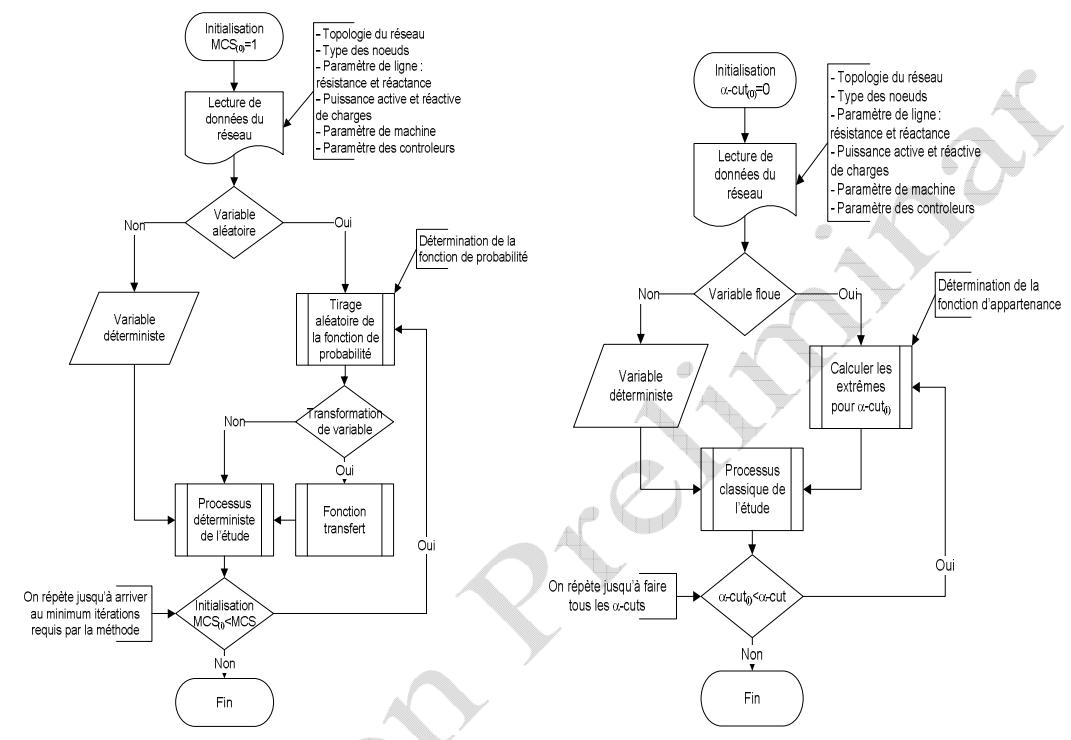

Figure 13. Algorithmes de: (a) Monté Carlo et (b) l'arithmétique floue.

\section{Description du réseau de distribution de test}

Le réseau de distribution «28-bus test » est présenté dans la référence (Khodr et al., 2006). Le système original est un système réel de distribution en $11 \mathrm{kV}$ (puissance de référence de 100kVA) auquel on a ajouté, sur le nœud 6, une source éolienne de $20 \%$ de la charge totale. Dans ce système, tous les nœuds sont des nœuds de type consommateur (PQ) à l'exception du nœud 1 qui est un nœud bilan avec une tension de nœud de 1 p.u. Le diagramme unifilaire du réseau est montré dans la figure 14 . 


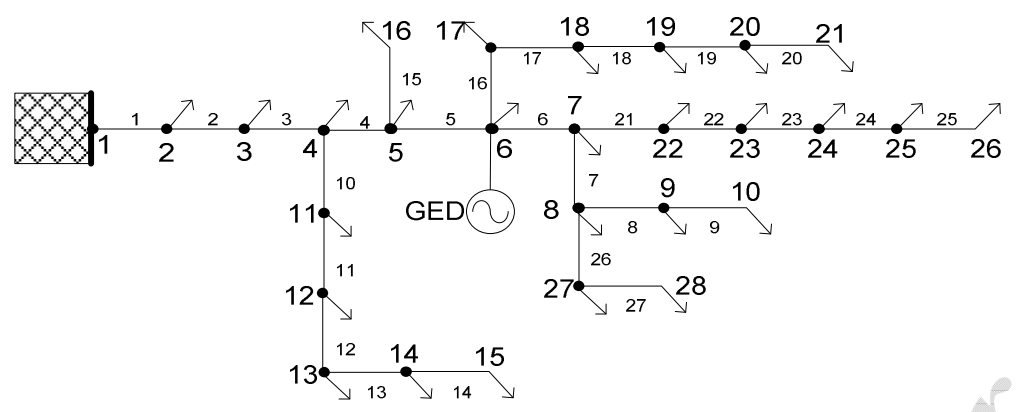

Figure 14. Diagramme unifilaire du réseau 28 nœuds.

Ce modèle suppose que le système triphasé équilibré peut être représenté par son équivalent monophasé direct. Dans le cas déséquilibré, les trois phases a, b, c doivent être prises en compte. Les données des paramètres électriques du réseau et charges pour le cas équilibré et déséquilibré sont indiquées dans l'annexe. Le modèle comprend les éléments suivants :

- Les lignes de distribution représentées par leurs résistances et réactances directes. Les capacités shunts des lignes sont négligées pour le réseau distribution.

- Les charges sont représentées par leurs puissances active et réactive.

- Le nœud de raccordement de l'éoliénne est considéré comme un nœud PQ d'injection de puissance avec un facteur de puissance inductif de 0,95.

Les paramètres d'incertitude sont représentés par une fonction de densité probabilité ou par des nombres flous. Ces deux modèles sont équivalents grâce au principe de cohérence de transformation de probabilité en possibilité avec une perte minimale d'information. Les nombres flous peuvent avoir une variété de formes (dans certaines conditions), mais par souci de simplicité, on traite principalement des données représentées par des fonctions d'appartenance trapézoïdale.

Les valeurs $a_{2}$ et $a_{3}$ de la fonction d'appartenance floue équivalente de chaque variable a été obtenue en appliquant transformation possibilité-probabilité de l'équation (11), en choisissant une valeur arbitraire de $S$, par exemple $S=0,05$ ainsi la condition $C$ supérieure ou égale à $2^{-1 / 2}$ soit accompli, dans ce cas $C=0,89$. Tandis que les valeurs $a_{1}$ et $a_{3}$ transformés à partir d'une fonction de probabilité gaussien ont relation avec la variance $(\sigma)$, du que la plus part de valeurs possibles de la variable $95.4 \%$ sont obtenus dans lintervalle $\pm 2 \sigma$ autour de la moyenne $(\mu)$, comme c'est montré dans la figure 1.a. Les sources d'incertitude prise en compte pour le calcul de répartition de charges sont attribuées aux éléments suivants :

- La puissance active et réactive des charges varie d'une manière continue en fonction d'une loi normale déterminée dans la section 6.3. L'incertitude de la 
distribution normale est caractérisée par $\sigma=8,51 \%$. Les extrêmes de la fonction floue trapézoïdale associée sont définis par le quadruple $\left(a_{1} ; a_{2} ; a_{3} ; a_{4}\right)=(0,90 ; 0,95 ; 1,05 ; 1,10)$ avec une valeur de $\mathrm{S}=0,05$ qui fait $\mathrm{C}=0,89$.

- Les paramètres électriques des lignes varient en fonction de la température suivant les conditions météorologiques et la charge. L'incertitude des lignes suit comme la charge une fonction normale de paramètre $\sigma=1,00 \%$ par rapport à la valeur nominale des valeurs de la ligne. Les extrêmes de la fonction trapézoïdale floue sont définis par le quadruple $\left(a_{1} ; a_{2} ; a_{3} ; a_{4}\right)=(0,98 ; 0,99 ; 1,01 ; 1,02)$ avec une valeur de $S=0,01$ qui fait $C=0,95$.

- La tension du poste source est régulée avec un changeur de prise en charge du transformateur de pas $\pm 2,5 \%$ pour maintenir la tension du réseau dans l'intervalle permis. L'incertitude de la tension du noud bilan est considérée comme une fonction normale avec $\sigma=1,25 \%$ par rapport à la valeur nominale des paramètres. Les extrêmes de la fonction floue trapézoïdale associée sont définis par le quadruple $\left(a_{1} ; a_{2} ; a_{3} ; a_{4}\right)=(0,975 ; 0,99 ; 1,01 ; 1,025)$ avec une valeur de $\mathrm{S}=0,01$ qui fait $\mathrm{C}=0,95$.

- La puissance produite par l'éolienne varie en fonction de la vitesse du vent. Les modèles d'incertitude probabiliste et possibiliste sont continus et correspondent aux modèles déterminés dans la section 6.4.3.

On considère que toutes les variables d'incertitude sont continues. L'intervalle de confiance des valeurs possibles d'incertitude floue est déterminé avec les alphacoups des fonctions d'appartenance $\mu_{A}(x)$ de chaque variable.

\section{Résultats obtenus}

Avant de considérer l'impact de toutes les variables entachées d'incertitude dans la répartition de charges, l'effet de la production décentralisée sur le profil de tension a été étudié de manière déterministe, ainsi que les pertes du réseau électrique. Les résultats déterministes du profil de tension et des pertes Joules du réseau avec et sans production décentralisée (GED) sont montrés dans la figure 15.
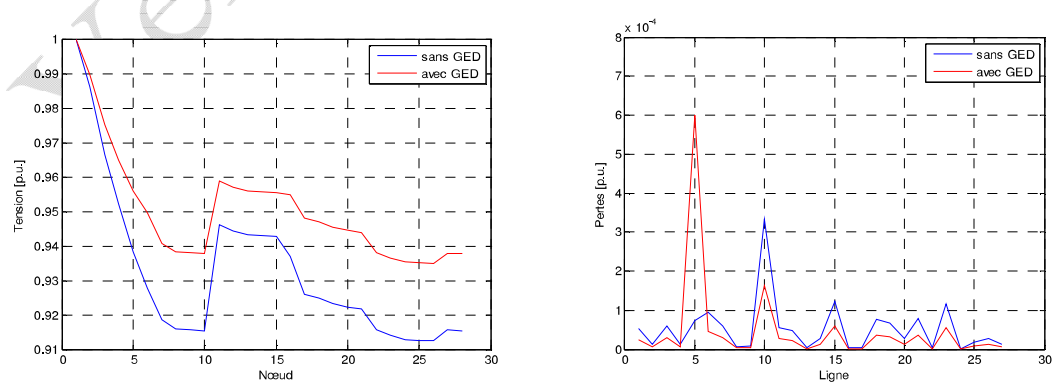

Figure 15. (a) Profil de tension et (b) de pertes de ligne du réseau 28 bus test. 
On observe que la chute de tension est moins grande après l'insertion de l'éolienne et que le profil de tension est amélioré dans les nœuds en amont et en aval de l'éolienne. Ce changement dans le profil de tension indique que la puissance est bien fournie par l'éolienne. De même, dans la figure 15(a), le profil des pertes par ligne du réseau est globalement plus bas pour le cas avec de l'éolienne compte tenu de son dimensionnement modeste, les pertes totales du réseau sans l'éolienne sont 1.88 fois de celles obtenus avec l'éolienne. On constate, pour ce cas, les aspects positifs de l'insertion de sources de l'éolienne au réseau de distribution. Cependant, il est à préciser que les pertes peuvent baisser ou augmenter et les surtensions peuvent survenir dans le réseau à la suite d'insertion de GED en fonction de taux de pénétration des GED, du lieu de raccordement, de l'état de charge, du type du réseau, entre autres.

Les résultats montrés dans la section suivante correspondent aux études de répartition de charges utilisant la méthode de l'arithmétique floue et la méthode de simulation de Monté Carlo. Les résultats obtenus par la simulation de Monté Carlo ont été faits pour 2500 tirages aléatoires avec une erreur marginale de 0,0475 et un degré de confiance de $99,9 \%$. La méthode de simulation de Monté Carlo représente la simulation de référence à comparer avec les résultats obtenus par la méthode floue. Les résultats des variables électriques (représentées par quatre courbes) obtenues par la méthode de l'arithmétique floue correspondent aux valeurs extrêmes d'incertitude du quadruple d'un nombre flou trapézoïdale. Les valeurs extrêmes du nombre flou des résultats sont comparées avec les résultats pour 2500 tirages aléatoires de la méthode de Simulation de Monté Carlo. On étudiera ainsi l'influence de l'incertitude.

Le premier cas de l'étude du réseau correspond à l'impact de la variation de la tension du poste source (avec une incertitude variable de 0.975 à 1.025 p.u.) sur le profil de tension. Les résultats sont représentés dans la figure 16 . On observe que les baisses de tension les plus significatives se trouvent sur les nœuds 10, 26 et 28 qui sont localisées après la GED dans les branches extrémités. Ces tensions nodales atteignent jusqu'à 0.90 p.u. pour les simulations de Monté Carlo tandis que par l'arithmétique floue elles sont plus basses. On observe, sur cet exemple, que l'incertitude de la tension est plus importante pour les résultats obtenus par la méthode de l'arithmétique floue. La GED injecte puissance, réduit la puissance consommée par le poste source et améliorer le profil de tension. En principe, l'effet de l'incertitude sur la chute de tension dépend principalement de la topologie et des valeurs relatives entre les charges et les impédances de ligne. Pour des lignes du réseau ayant les mêmes paramètres électriques $(\Omega / \mathrm{Km})$, l'incertitude dépendra de la longueur de la ligne définie par la topologie du réseau et de la température.

Les charges équivalentes vues par les nœuds 10, 26 et 28 sont les plus grandes du réseau (ce sont des extrémités des lignes), en effet l'intervalle de variation de chute de tension est plus grand sur ces nœuds que sur les autres. La variation de tension sur les nœuds 28 et 26 est de $4.25 \%$; elle est de $4.15 \%$ pour le nœud 10 . Quand on observe les résultats obtenus par Monté Carlo, on voit que très peu de valeurs s'approchent des valeurs extrêmes obtenues par la méthode de l'arithmétique 
floue. Ces valeurs correspondent au tirage aléatoire avec une faible probabilité d'occurrence.
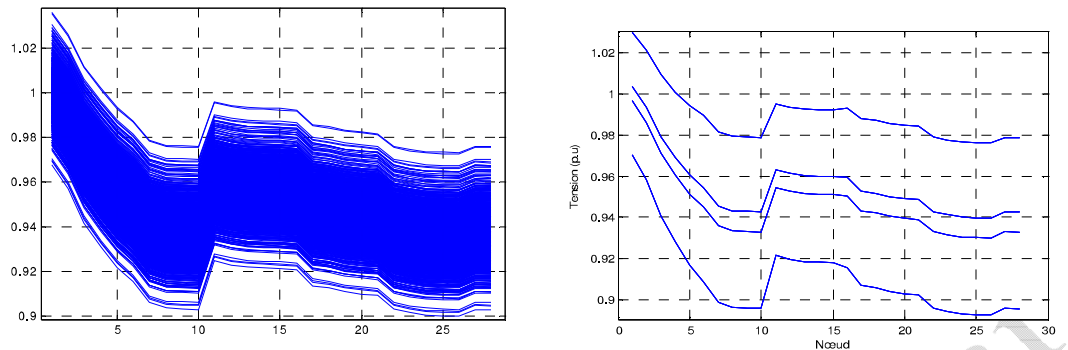

Figure 16. Profil de tension avec incertitude sur la tension de la source par (a) la méthode de Monte-Carlo (b). la méthode floue

La figure 17 montre le profil de tension avec la prise en compte de l'incertitude uniquement sur les paramètres des lignes. Dans le profil de tension déterminé par la méthode de Monté Carlo, la tension baisse pour le nœud 26 (le point plus bas) jusqu'à 0.93 p.u. Les variations les plus importantes se trouvent sur les nœuds 10, 26 et 28 comme dans le cas précèdent. Le profil de tension donné par la méthode floue contient les valeurs extrêmes de tension. Donc, l'arithmétique des intervalles floue trouve la variation de tension avec moins de points que la simulation de Monté Carlo. La variation de tension extrémale sur les nœuds 28,26 et 10 est respectivement de $1.06 \%, 0.8 \%$ et $1 \%$ dans le cas possibiliste tandis qu'elle est de $0.98 \%, 0.76 \%$ et $0.95 \%$ dans le cas probabiliste.
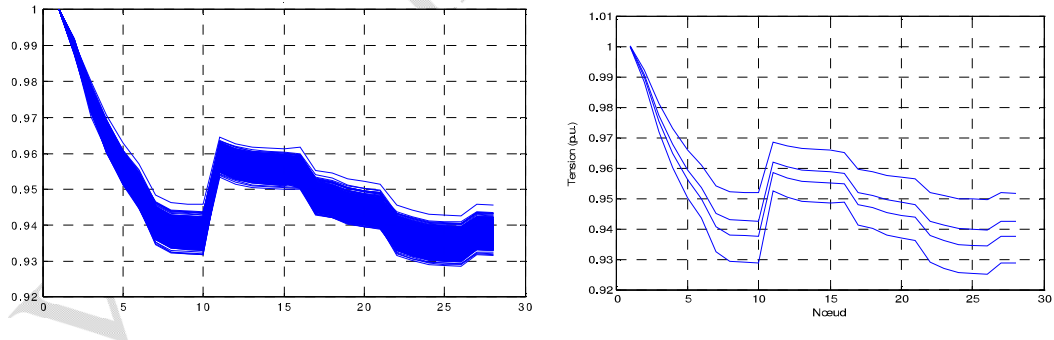

Figure 17. Profil de tension avec incertitude sur les lignes par (a) la méthode de Monte-Carlo (b) la méthode floue

La figure 18 montre le profil de tension avec incertitude sur les charges. On observe que la chute de tension est plus grande dans ce cas (en arrivant jusqu'à 0.91 p.u) par rapport au cas de variation des paramètres de ligne (avec une chute de tension jusqu'à 0.93 p.u). Ainsi, l'impact de la charge est plus grand que celui des paramètres de lignes avec les variabilités considérées. Les valeurs obtenues par 
l'arithmétique floue sont plus grandes que celles obtenues par la simulation de Monte-Carlo, de nouveaux, elles contiennent les résultats. La variation de tension dans le cas possibiliste sur les nœuds 28 et 10 est respectivement de $1.61 \%$ et $1.7 \%$, tandis que pour le cas probabiliste elle est respectivement de $1.16 \%$ et $1.17 \%$.
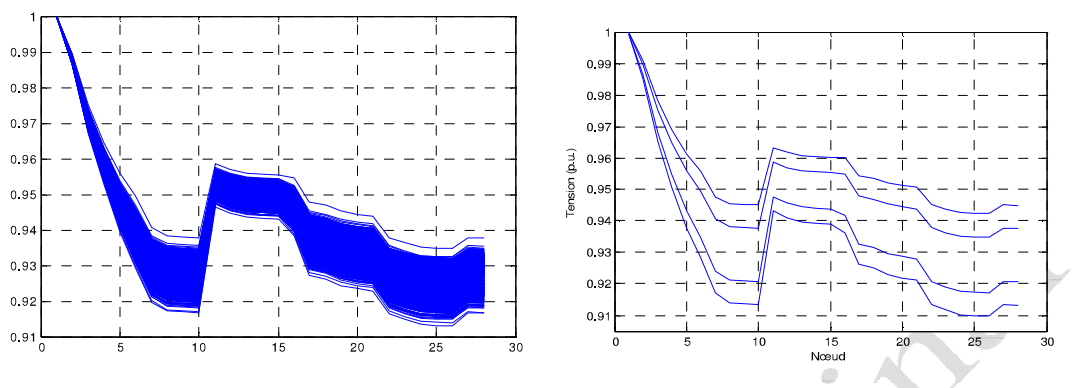

Figure 18. Profil de tension avec incertitude sur les charges par (a) la méthode de Monte-Carlo (b) la méthode floue

La figure 19 montre le profil de tension avec incertitudes sur, simultanément, la tension de la source, les paramètres des lignes et les charges. La variation de tension sur les nœuds 28 et 10 dans le cas possibiliste est respectivement de $4.81 \%$ et $5 \%$, tandis que dans le cas probabiliste elle est respectivement de $3.62 \%$ et $3.74 \%$. Le temps de simulation avec un processeur Intel-core i5 $1333 \mathrm{MHz}$ et $5-\mathrm{Gb}$ par la méthode de Monte-Carlo a été de 22 secondes tandis que pour la méthode floue, il a été uniquement de 0.87 secondes. Les résultats de l'arithmétique floue sont plus larges pour les limites supérieures que pour les limites inférieures, mais les tendances des résultats sont assez semblables.
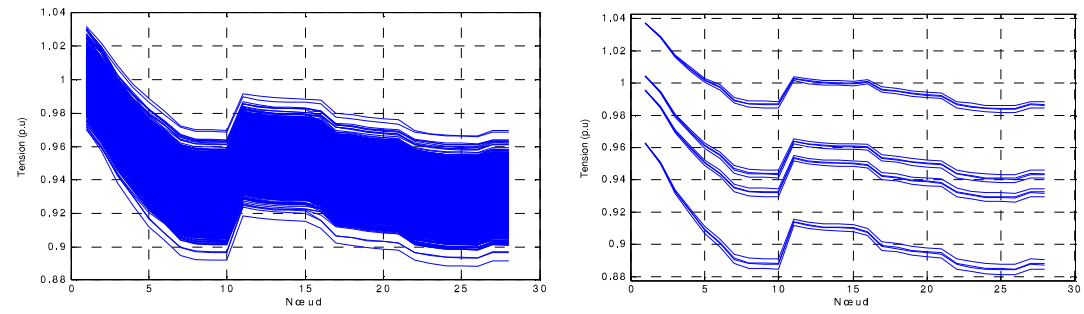

Figure 19. Profil de tension tenant compte l'incertitude de tension de la source, des paramètres de lignes et des charges par (a) la méthode de Monte-Carlo (b) la méthode floue

On a aussi calculé les pertes par effet Joule des différentes lignes du réseau de distribution pour comparer les résultats des deux méthodes dans la figure 20. On peut voir que l'estimation faite par la méthode de l'arithmétique floue est plus 
grande dans la plage des valeurs de pertes occasionnées par les différences dans la chute de tension et l'erreur quadratique entre les deux méthodes. De la même manière que pour les autres cas, les limites d'incertitudes obtenues par l'arithmétique floue sont plus importantes que pour la méthode de Monte-Carlo. La variation la plus importante est observée au niveau de la branche 5 entre les nœuds 5 et 6 où l'éolienne est couplée. Le pic de pertes sur la branche 5 est causé par un refoulement de puissance vers l'amont.
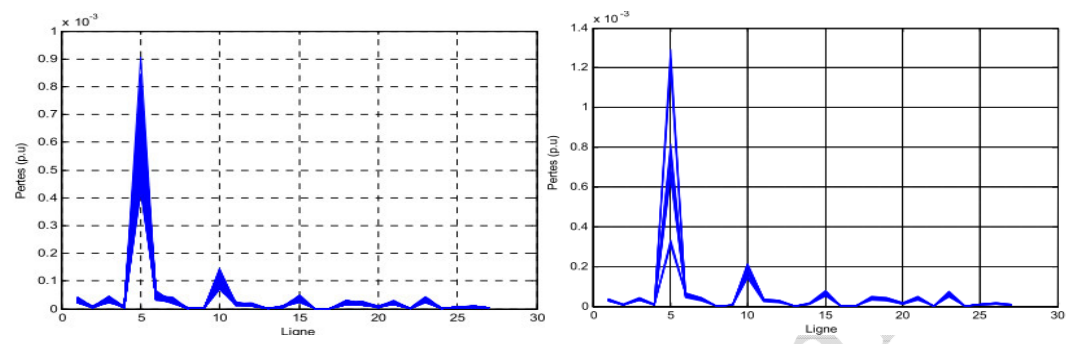

Figure 20. Profil pertes tenant compte l'incertitude de tension de la source, des paramètres de lignes et des charges par

(a) la méthode de Monte-Carlo (b) la méthode floue

On peut remarquer, sur le temps de convergence déjà mentionné pour les deux méthodes, que la méthode de Monte Carlo est plus lente que l'arithmétique floue (25 fois le temps requis dans ce réseau de 28 nœuds) pour obtenir une erreur marginale de 0,0475 et un degré de confiance de $99,9 \%$. Le temps de convergence d'une méthode est très important pour les calculs associés à un réseau plus grand (plus de nœuds) par rapport à une autre méthode. Du fait que la méthode de l'arithmétique floue a été plus rapide dans l'évaluation des incertitudes que la méthode de simulation de Monté Carlo ; on a poursuivi l'évaluation et mis au œuvre des deux méthodes dans les études statiques du réseau électrique.

\section{Conclusion}

Dans le calcul de répartition de charges, les variables électriques sont traitées normalement de manière déterministe, considérant des valeurs comme la prédiction de la charge. L'intégration des GEDs dans les réseaux de distribution fait que la prise en compte uniquement des valeurs déterministes extrémales, dans les calculs, pourrait sous-estimer les résultats de simulation et conduire à des conclusions trop pessimistes. Une alternative est de considérer la combinaison des variables dans les algorithmes comme un ensemble incertain. Le modèle des variables électriques peut être construit aussi de manière stochastique en prenant compte des incertitudes pour l'évaluation de son impact. Les modèles d'incertitude sont fondés soit sur l'utilisation directe des résultats de mesures, soit sur le choix d'une distribution de 
probabilité empirique adaptée aux valeurs mesurées (approche probabiliste) ou soit avec distribution de possibilité (approche floue).

Les variables d'incertitude analysées ont été principalement la demande et la production décentralisée. La demande des clients est un paramètre qui change considérablement au cours de la journée, la semaine et l'année. La production d'énergie des éoliennes varie aussi en fonction des conditions météorologiques. Ces deux facteurs causent des difficultés dans l'estimation des pertes de puissance par exemple. D'autres types d'incertitudes ont été considérés dans les études de ce chapitre, comme des petites variations des paramètres de ligne (liées par exemple à une dépendance à la température) et de la tension du poste source (liée à des variations de tension du réseau amont par exemple). Ces modèles d'incertitude servent à évaluer l'impact de l'incertitude et les exigences du comportement des variables électriques du réseau.

La mise en œuvre des approches probabiliste et possibiliste a été testée dans le cadre d'un calcul de répartition des charges. L'approche probabiliste est mise en œuvre en appliquant la méthode de simulation de Monté Carlo et la modélisation des incertitudes des variables électriques (puissance, paramètre de ligne, entre autres) est réalisée avec des fonctions de densité de probabilité. L'approche possibiliste est faite en appliquant la méthode de l'arithmétique des intervalles floue et la modélisation des incertitudes est faite avec des fonctions d'appartenance floues. Les nombres flous associent un degré d'appartenance de chaque valeur possible de la variable. On peut obtenir la fonction d'appartenance floue à partir des données de mesures ou des erreurs de mesures (Briceno Vicente, 2012).

Les résultats des études menées sur les réseaux test ont montré que l'intervalle de variation des sorties par la méthode de l'arithmétique floue contient celles obtenues par la méthode de Monte-Carlo. La méthode de l'arithmétique floue a aussi une meilleure performance au niveau de temps de calcul par rapport à la méthode de Monte-Carlo.

La raison de la différence entre les résultats de deux méthodes est que l'arithmétique des intervalles flous utilise les valeurs extrêmes des variables (minimum et maximum) dans les opérations arithmétiques. Par contre, la méthode de simulation de Monté Carlo fait un tirage aléatoire pour chaque variable d'incertitude en fonction de sa probabilité d'occurrence. La méthode de simulation de Monte-Carlo n'explore pas les pires valeurs de faible probabilité ; cela oblige le lancement d'un grand nombre d'itération pour obtenir une loi normale, ce qui augmente le temps de calcul. Le temps de simulation peut devenir significatif pour des réseaux de grande taille.

La méthode de l'arithmétique floue peut être utilisée dans les réseaux plus de plus grande dimension (grand nombre de variables incertaines), où l'application de la méthode de Monte-Carlo prendrait trop de temps. Le fait qu'il soit possible de convertir des nombres flous en des fonctions de densité de probabilité permet d'évaluer indirectement les probabilités en termes de fonctions de distribution de possibilité. Il est important de noter que la fonction d'appartenance doit satisfaire les conditions de base mentionnées dans la section 4.3 afin d'appliquer la 
transformation. Aussi, pour l'application de la méthode de l'arithmétique il faut que l'algorithme de base pour la résolution du problème soit défini en termes d'opérations arithmétiques simples, voir section 4.6. Le temps de simulation évité par la méthode de l'arithmétique floue par rapport à la simulation de Monté Carlo dépend aussi de la méthode de résolution de l'algorithme de base. Ces deux méthodes (probabiliste et possibiliste) peuvent être appliquées dans n'importe quelle topologie du réseau avec la limite des opérations arithmétiques simples.

La méthode de l'arithmétique floue est utile afin d'évaluer les incertitudes, liées à l'intégration des sources d'énergie stochastiques qui ont un impact plus fort que la production d'énergie conventionnelle dans les études du réseau en raison de leur production d'énergie variable (Briceno Vicente, 2012).

\section{Bibliographie}

Alberta Electric Operator System (AESO). 10 Minute Historical Data for Total Wind Power and Alberta Internal Load. Wind Power / AIL Data., 11 Oct. 2011.

Allan R., Al-Shakarchi M., Probabilistic ac load flow, Electrical Engineers, Proceedings of the Institution of, vol. 123, n 6 , p. 531-536, 1976.

Allan R., Al-Shakarchi M., Linear dependence between nodal powers in probabilistic ac load flow, Electrical Engineers, Proceedings of the Institution of, vol. 124, n 6, p. 529-534, 1977.

Allan R., Borkowska B., Grigg C., Probabilistic analysis of power flows, Electrical Engineers, Proceedings of the Institution of, vol. 121, $\mathrm{n}^{\circ} 12$, p. 1551-1556, 1974.

Allan R., Leite da Silva A., Burchett R., Evaluation methods and accuracy in probabilistic load flow solutions, power apparatus and systems, IEEE transactions on, $\mathrm{n}^{\circ}$ 5, p. 2539-2546, 1981.

Alvarado F., Hu Y., et Adapa R., Uncertainty in power system modeling and computation, in IEEE International Conference on Systems, Man and Cybernetics, vol. 1, 1992, pp. 745-760.

Anders, G. J., Probability concepts in electric power systems, John Wiley and Sons Inc.1989.

Bloom J., Probabilistic production costing with dependent generating sources, Power Apparatus and Systems, IEEE Transactions on, n 8, p. 2064-2071, 1985.

Borkowska B., Probabilistic load flow, Power Apparatus and Systems, IEEE Transactions on, $\mathrm{n}^{\circ}$ 3, p. 752-759, 1974.

Briceño Vicente, W. C. Modélisation des réseaux de distribution sous incertitudes (Doctoral dissertation, Université de Grenoble, Grenoble). Septembre 20, 2012 
Caramanis M., Tabors R., Nochur K., Schweppe F., The Introduction of NonDIispatchable Technologies a Decision Variables in Long-Term Generation Expansion Models, Power Apparatus and Systems, IEEE Transactions on, $\mathrm{n}^{\circ} 8, \mathrm{p}$. 2658-2667, 1982.

Civanlar M. R., Trussell H. J., Constructing Membership Functions Using Statistical Data, Fuzzy Sets and Systems., Vol. 18, 1986, pp. 1-13.

Council G., Perpectivas Globales de la Energía Eólica, 2006.

Dopazo J., Klitin O., Sasson A., Stochastic load flows, Power Apparatus and Systems, IEEE Transactions on, vol. 94, n 2, p. 299-309, 1975.

Dubois D., Prade H., Fuzzy Sets and Probability: Misunderstandings, Bridges and Gaps, Second IEEE International Conference on Fuzzy Systems, vol.2, pp. 1059-1068, 1993

Dubois D., Prade H., Sandri S., On Possibility/Probability Transformations, Fuzzy Logic, Kluwer Academic Publishers, pp.103-112, 1993.

Ellard D., Ellard P., SQ Course Book, 2003

Hanss M., Applied fuzzy arithmetic, Springer, 2005.

Khodr H., Ocque L., Yusta J., Rosa M., New Load Flow Method SE Oriented For Large Radial Distribution Networks, Transmission \& Distribution Conference and Exposition : Latin America, 2006. IEEE/PES, IEEE, p. 1-6, 2006.

Klir G., Yuan B., Fuzzy sets and fuzzy logic : Theory and Applications, Possibility Theory versus Probability Theory, Prentice Hallp. 200-207, 1995.

Kundur, Prabha. Power system stability and control. Eds. Neal J. Balu, and Mark G. Lauby. Vol. 7. New York: McGraw-hill, 1994.

Leite da Silva A., Allan R., Soares S., Arienti V., Probabilistic load flow considering network outages, Generation, Transmission and Distribution, IEE Proceedings C, vol. 132, IET, p. 139-145, 1985.

Leite da Silva A., Arienti V., Probabilistic load flow by a multilinear simulation algorithm, Generation, Transmission and Distribution, IEE Proceedings C, vol. 137, IET, p. 276-282, 1990.

Leite da Silva A., Arienti V., Allan R., Probabilistic load flow considering dependence between input nodal powers, power apparatus and systems, ieee transactions on, $n^{\circ} 6$, p. 1524-1530, 1984.

Sauer P., Generalized stochastic power flow algorithm, Technical report, Purdue Univ., Lafayette, IN (USA). School of Electrical Engineering, 1977.

Teng J., A direct approach for distribution system load flow solutions, Power Delivery, IEEE Transactions on, vol. 18, $\mathrm{n}^{\circ} 3$, p. 882-887, 2003.

Zadeh L., Fuzzy sets as a basis for a theory of possibility, Fuzzy sets and systems, vol. 100, p. 9-34, 1999. 


\section{Annexe. Le réseau de distribution « 28 bus test »}

Les données du réseau du réseau de distribution (Khodr et al., 2006) de la section 7 et du diagramme unifilaire de la figure 14 sont le tableau suivant avec des bases de tension et de puissance de $11 \mathrm{kV}$ et $100 \mathrm{kVA}$.

Tableau 3. Les paramètres de lignes du réseau de distribution 28 nœuds.

\begin{tabular}{|c|c|c|c|c|c|}
\hline \multirow[b]{2}{*}{$\begin{array}{c}\text { Ligne } \\
\mathrm{De}-\mathrm{A}\end{array}$} & \multicolumn{2}{|c|}{ Paramètre de ligne } & \multirow[b]{2}{*}{$\begin{array}{c}\text { Ligne } \\
\mathrm{De}-\mathrm{A}\end{array}$} & \multicolumn{2}{|c|}{ Paramètre de ligne } \\
\hline & $\begin{array}{c}\text { Résistance } \\
\text { [p.u.] }\end{array}$ & $\begin{array}{l}\text { Réactance } \\
\text { [p.u.] }\end{array}$ & & Résistance [p.u.] & $\begin{array}{c}\text { Réactance } \\
\text { [p.u.] }\end{array}$ \\
\hline $01-02$ & 0,00151 & 0,00063 & $15-16$ & 0,00227 & 0,00096 \\
\hline $02-03$ & 0,00184 & 0,00078 & $16-17$ & 0,00341 & 0,00064 \\
\hline 03-04 & 0,00113 & 0,00047 & $17-18$ & 0,00227 & 0,00064 \\
\hline $04-05$ & 0,00076 & 0,00031 & $02-19$ & 0,00284 & 0,00080 \\
\hline $05-06$ & 0,00301 & 0,00125 & $19-20$ & 0,00114 & 0,00032 \\
\hline $06-07$ & 0,00226 & 0,00094 & $20-21$ & 0,00227 & 0,00064 \\
\hline $07-08$ & 0,00120 & 0,00050 & $21-22$ & 0,00409 & 0,00116 \\
\hline $08-09$ & 0,00226 & 0,00094 & $03-23$ & 0,00296 & 0,00084 \\
\hline $09-10$ & 0,00301 & 0,00125 & $23-24$ & 0,00250 & 0,00071 \\
\hline $10-11$ & 0,00227 & 0,00064 & $24-25$ & 0,00455 & 0,00129 \\
\hline $11-12$ & 0,00114 & 0,00032 & $06-26$ & 0,00227 & 0,00064 \\
\hline $12-13$ & 0,00341 & 0,00096 & $26-27$ & 0,00114 & 0,00032 \\
\hline $13-14$ & 0,00341 & 0,00071 & $27-28$ & 0,00114 & 0,00032 \\
\hline $14-15$ & 0,00250 & 0,00064 & $Y$ & & \\
\hline
\end{tabular}

